\title{
Implementation of Virtual Visualization in Mountain Scenic Spots Based on Spatial Integration Technology
}

\author{
Sisi $\mathrm{Lv}^{1}$, Weiquan $\mathrm{Zhao}^{2, *}$, Wei $\mathrm{Li}^{2}$ and Weici $\mathrm{Su}^{2}$ \\ ${ }^{1}$ School of Karst Science, Guizhou Normal University, Guiyang, Guizhou 550001, China \\ ${ }^{2}$ Institute of Mountain Resources of Guizhou Province, Guiyang, Guizhou 550001, China \\ ${ }^{*}$ Corresponding author
}

\begin{abstract}
With the development of the tourism, tourism virtual visualization became the essential method to show the charm of scenic spots. Study on the integration technology of panoramic images, 3D GIS and cloud GIS to visualize the mountain scenic spots in order to meet the needs of travelers, the integration technology supports real feel about site, adds an online travel manner. The advantages of cloud GIS are reducing the development cost, shortening the development cycle, integrating multi-data (space and non-space data) in the open style database. The characteristics of the mountain scenic, like terrain ups and downs, different scenes in seasons and seasons' scenery in mountains are vividly showed by the virtual visualization system. The system provides tour with a new direct interactive abundant feel.
\end{abstract}

Keywords-cloud GIS; vitual visualization; panoramic images; 3D GIS

\section{INTRODUCTION}

With the rapid development of tourism industry, the original natural landscape, the rich natural and cultural tourism resource, terrain ups and downs, the special landscape of "different scenes in seasons and seasons' scenery in mountains" attract more and more people. Breaking through the traditional way can show the mountain tourism resource to people, which is a serious subject. The way of tourism has been changing through the evolution of the internet and visualization techniques. At the beginning, the managers of scenic spot published tourist information to attract visitors to promote economic development in the scenic area by using the texts, pictures and videos. $\mathrm{Xu}$ [6]and Guan [7] explored the tourist website framework. Then, with the Geographic Information System (GIS) development and the cloud GIS proposition, GIS is widely used in the tourism application, many scholars propose a tourism approach based on GIS, for example, Cao [1] and Wirjono [2] described the GIS application in Hainan and Indonesia tourism; Dong [8], Zhao [9] used Web GIS to establish the tourist information system, Marcus designed the mobile GIS using[3]. Virtual tourism was putted forward by Hobson and Williams [4] in 1995 and came into the popular sight gradually; Yang [10] summarized the development level of virtual visualization in China. There are two main virtual ways; one is the panorama, for instance, $\mathrm{Lu}$ [11] explored the panorama in her research; the other one is three-dimension (3D) technique, such as, Helmut Berger [5] pointed out the new pattern of electronic tourism (e-tourism) with 3D technique; Zhang [12] designed the application of the virtual technique on the tourism system based on the Web GIS; Jiang [14], Wei [15] and Gao [15]used the 3D model features and landscapes in sites. The using of visual and GIS technique not only bring the convenience to travelers, but also attract potential customers. Virtual visualization realized modeling tourism resources stands out features of the mountain scenic spots, like geomorphology, cultural and natural landscape. We use the integration technology of panorama/3D GIS/cloud GIS to support the tourism resource visualization, these techniques are beneficial for each other in aspects of spatial analysis and spatial display, which makes online tourism experience more real.

\section{Key TECHNOLOGIES}

\section{A. Panorama}

Panorama, called panoramic photography or panoramic images, is the virtual reality technology based on static images, but it's not the real 3D. Photographers take assembling multiple images of a view into a single wide image. The technique makes "spherical" panorama movies (that incorporate a full $180^{\circ}$ vertical viewing angle as well as $360^{\circ}$ around) by stitching multiple images. In contrast with 3D technology, panorama images of data acquisition and processing are simpler and bring customers true feelings. The panoramic operations (zoom in, zoom out and rotate) have powerful interchange with users, and give visitors more real experience comparing with the traditional photograph.

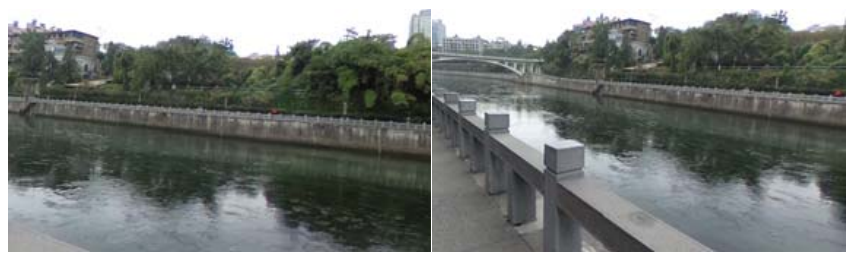

FIGURE I. PANORAMIC IMAGES FROM DIFFERENT VIEW

\section{B. 3D GIS}

3D GIS is the extension of the GIS and 3D technology, surface features can be modeled on the basis of geographic data and pictures, the GIS space analysis also can be used. In $3 \mathrm{D}$ scene, the topological relation between features is clearer than that of the $2 \mathrm{D}$; the simulate scenes of scenic spots give travellers more information about tourist resource, especially in natural and cultural landscape. 


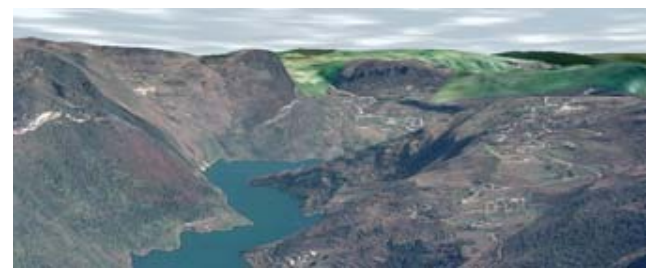

FIGURE II. TOPOGRAPHY IN 3D SENCE

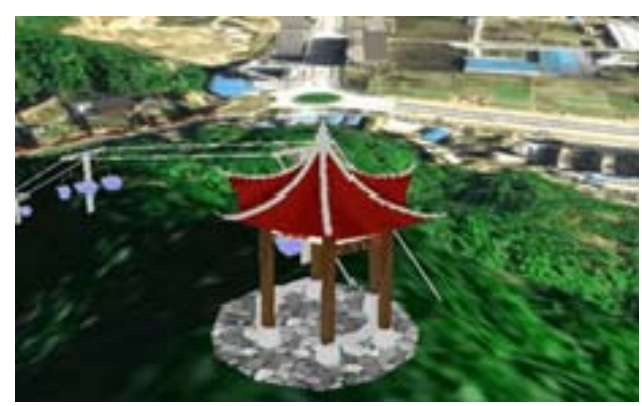

FIGURE III. CULTURAL FEATURE MODEL

\section{Cloud GIS}

Cloud GIS means that the elements of geospatial information can be putted into the cloud, including data, processing, modeling, and so on. Cloud computing could make it possible to administrate the on-line data and analysis processing, which opens a new low-cost friendly dimension for GIS management. According to their own requirements, users effectively choose geographic information resources (data, function, and model) in the cloud (public cloud or private cloud). It has change traditional GIS application and development mode and is the necessary vehicle of GIS data shared services.

\section{INTEGRATION DESIGN}

\section{A. Iintegrated Application Framework}

The system framework (Figure IV) has four parts: users' level, service management level, resource pool and infrastructure level. It must be clear who is the system user and what are his demands at the users' level. Service management level keeps system running, regulates the resource use, including the log, deployment and state of system. Geo-spatial data, no-spatial data, analysis services and panorama resource stored in the resource pool are the system core. Infrastructure level contains the software and hardware, software includes operating system, skyline software, ArcGIS, stitching software, data base management system(DBMS), different software has different duties; hardware contains storage, network devices, data acquisition tools(e.g., panoramic camera and scanner). Skyline is used for building the 3D scenes. Using ArcGIS edits to manage spatial data and publish different type geographical map service in cloud. DBMS interacts with the user, allows users to create, maintain and manipulate no-spatial data. The system also must follow specification standards, such as shape format, XML and database design standard group into data standard; dynamic web pages written specifications include HTML, CSS, Asp.net,
JavaScript; data communication standard has the representational state transfer (REST) application programming interface (API) and open database connectivity (ODBC) specification, otherwise, stitching images rules and software design specifications exist in specification standards.

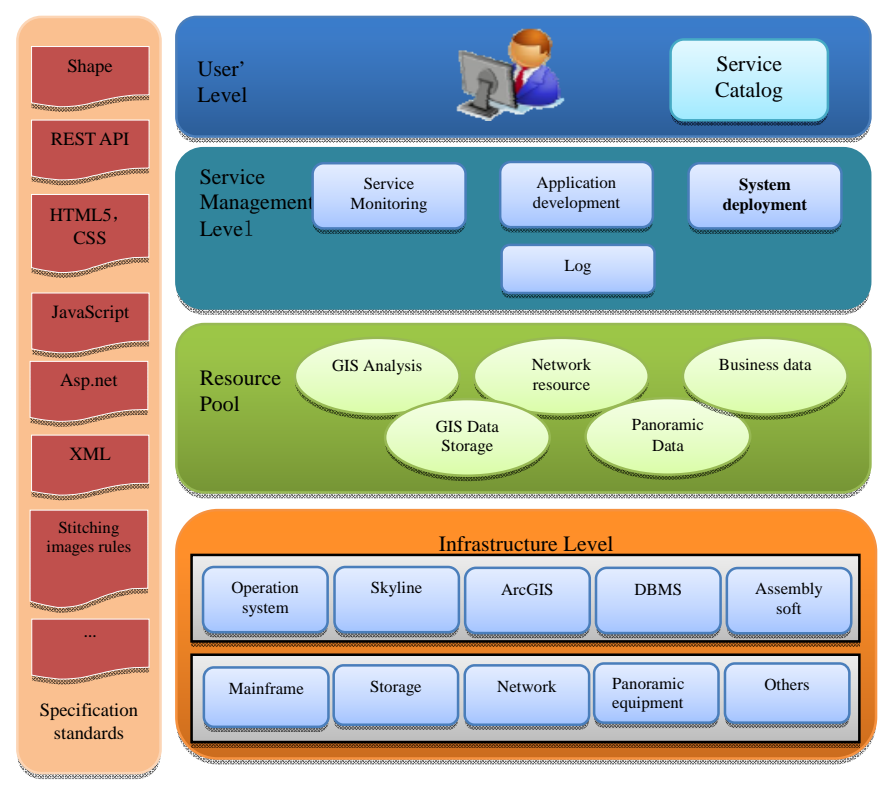

FIGURE IV. THE SYSATEM FRAMEWORK

\section{B. Data and Integrated Application}

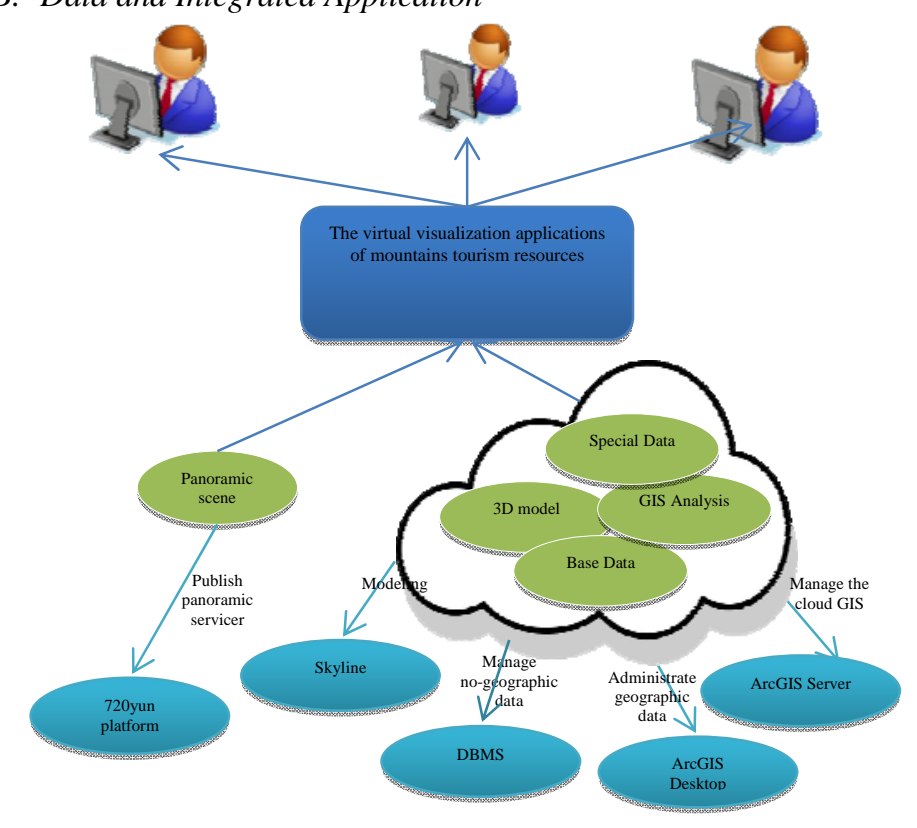

FIGURE V. DATA MANAGEMENT STRUCTURE

Four different data managements are supported by different platforms. First, 3D data, the administrator can establish humanity moldings or vegetation ecological landscape on 3D terrain based on skyline. Second, geographic data and spatial analysis, open cloud networking of ArcGIS allows for effective utilization of the GIS resource, using 
ArcGIS desktop manage and edit geographic data in the cloud, using ArcGIS server monitor and maintain the state of GIS resource. Third, panoramic images, we can publish images as panoramic services on the website (720yun). Four, business data (e.g., scenic spot news, shops information, service information), data features are constantly changing, real-time, elasticized and complicated relationship, DBMS should be selected to manage it. (Figure V)

The system development language: accessing and operating database make use of asp.net and ODBC on the basis of C\# language. Using the JavaScript implement the secondary development of skyline, call a REST API to visit panoramic services and geo-web services.

\section{Main Function Realized}

Skyline platform could make it possible to show the character of mountain scenic spots, but the simulation scene is too elegant to look like the real as other 3D technology. This research mixes panorama and $3 \mathrm{D}$ technology to bring the real feel to visitors, panoramic images compensate for the 3D weakness. The function is clicking scenic spots then getting the result of spatial query which is the panoramic service locator to show panoramic images in a pop-up box with the JavaScript API (SGWorld API) (Figure VI). The code to do this is shown below.

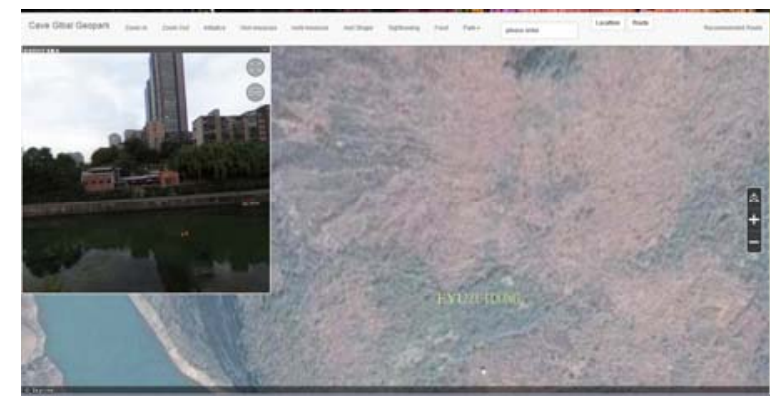

FIGURE VI. THE COMBINED UTILIZATION OF 3D AND PANORAMA

function OnLButtonDblClk(Flags, X, Y) \{

var url720 = null;

var ItemID = SGWorld.ProjectTree.FindItem("scenic spots");

var obj = SGWorld.ProjectTree.GetLayer(ItemID);

// click to get XY

var pos = SGWorld.Window.PixelToWorld(X, Y); var cVerticesArray $=[-122.415025,37.76059,10$,$] ;$ var qobj =

SGWorld.Creator.GeometryCreator.CreatePointGeom etry(cVerticesArray);

qobj.X = pos.Position. $X$;

qobj.Y = pos.Position. $Y$;

qobj.Z = pos.Position.Altitude;

var newGeo = qobj.SpatialOperator.buffer(50);

//SpatialQuery

var resFeatures = obj.ExecuteSpatialQuery(newGeo, $1)$;

for (var $\mathrm{i}=0$; $\mathrm{i}<$ resFeatures.Count; $\mathrm{i}++$ ) \{

var pIFeature $=$ resFeatures.Item(i); url720 = pIFeature.FeatureAttributes.Item(5).Value;

\}

var popup $=$

SGWorld.Creator.CreatePopupMessage("panorama", url720, 10, 10, 500, 500);

popup. Width $=3500$;

popup.height $=3500$;

SGWorld.Window.ShowPopup(popup);

\}

The navigation application is widely used in the tourism. The system highlights the advantage of cloud GIS and the dynamic effect of 3D display, getting a navigation path from cloud with the REST API and creating dynamic route view which can see the virtual scene around. Following codes which contain parsing the JSON result from ArcGIS for Server REST resource, rendering the route (Figure VII) and building the virtual way (Figure VIII).
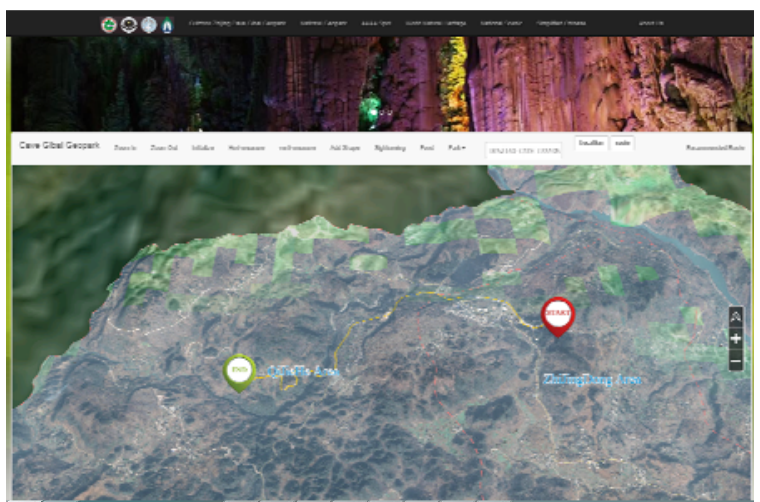

FIGURE VII. RESULT OF A RENDERING PATH

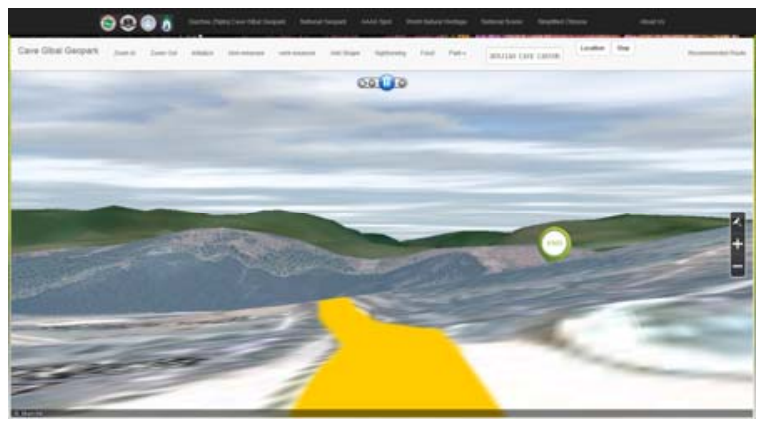

FIGURE VIII. DYNAMIC ROUTE

\$("\#gps_submit").click(function () \{

if (toolstatus $==0$ ) \{

\$(this).text("Route");

toolstatus $=4$;

//using IE object-geolocation get $\mathrm{XY}$ as the start point

startPosition=getLocation();

var startPosition = new Object();

startPosition. $\mathrm{x}=$ startPosition. $\mathrm{x}$;

startPosition.y =startPosition.y;

var endPosition = new Object();

endPosition. $\mathrm{X}=$ jditem.POINT_X;

endPosition.y = jditem.POINT_Y; 
//set RESR URL

var restURL = getURL(startPosition, endPosition);

//delete exiting dynamic route view

var group_id =

SGWorld.ProjectTree.FindItem("navigation");

if (group_id !=0) \{

SGWorld.ProjectTree.DeleteItem(group_id);

\}

var routeLocation $=[]$;

var group =

SGWorld.ProjectTree.CreateGroup("navigation");

var presentation $=$

SGWorld.Creator.CreatePresentation(group, "path");

presentation.PlaySpeedFactor $=3$;

presentation.PlayAlgorithm = 1 ;

//post REST request with ajax

\$.ajax $(\{$

type: "get",

url: restURL,

dataType: "json",

contentType: "application/json",

success: function (result) \{

// parse json

\$.each(result.routes, function(routes_Index,

routes_info)

\{

if (routes_Index $==$ "features") \{

\$.each(routes_info, function (features_Index,

features_info) \{

\$.each(features_info, function (Index, info) \{

if (Index == "geometry") \{

\$.each(info, function (geometry_Index, geometry_info)

\{

if (geometry_Index == "paths") \{

\$.each(geometry_info, function (path_Index, path_info) \{

\$.each(path_info, function (Index1, info1) \{

if (Index1 < path_info.length - 1) \{

//get presentation direction between two point

var coord $1=$

SGWorld.Creator.CreatePosition(path_info[Index1][0], path_info[Index1][1], 0);

var coord2 =

SGWorld.Creator.CreatePosition(path_info[Index1 +

1][0], path_info[Index $1+1][1], 0)$;

var angles = coord1.AimTo $($ coord2);

var yaw = angles. Yaw.toFixed(5);

var paths $=$ SGWorld.Creator.CreatePosition(info1[0],

info1[1], 10, 2, yaw, 0, 0, 0);

//add route paths

presentation.CreateLocationStep(1, 0, Index1, paths);

routeLocation.push(coord1);

\}

else \{

//set images of the start point and end point

var coord1 =

SGWorld.Creator.CreatePosition(info1[0], info1[1], 0);
routeLocation.push(coord1);

var labelStyle $=$ SGWorld.Creator.CreateLabelStyle(); var labelPos $=$

SGWorld.Creator.CreatePosition(startPosition.x,

startPosition.y, 0, 2);

var label1 = SGWorld.Creator.CreateLabel(labelPos,

"", toAbspath("pic/start.png"), labelStyle, group);

var labelPos =

SGWorld.Creator.CreatePosition(endPosition.x,

endPosition.y, 0, 2);

var label1 = SGWorld.Creator.CreateLabel(labelPos, "', toAbspath("pic/end.png"), labelStyle, group);

//build dynamic paths

var geometry $=$

SGWorld.Creator.GeometryCreator.CreateLineString

Geometry(routeLocation);

var color $=$ SGWorld.Creator.CreateColor(255, 204, 0, $0.7)$;

var line = SGWorld.Creator.CreatePolyline(geometry, color, 2, group, "my poly on terrain");

line.LineStyle. Width = 10;

SGWorld.Navigate.FlyTo(line);

\}\});\}); \}\}); \}); ;);\}\});\},

error: function (XMLHttpRequest, textStatus, errorThrown) \{

alert(XMLHttpRequest.status);

\}$\}) ;\}\})$;

\section{CONCLUSION}

Tourism virtual visualization has became the tourist tendency since information technology developed gradually. The research implements the virtual mountain tourist resource visualization with the integration technology of panoramic images, 3D GIS and cloud GIS, which standing out the mountain features (terrain ups and downs, the confluence of natural landscape and humanitarian landscape, the plant diversity, and so on). Virtual visualization satisfies the visitors' needs to strengthen the credibility of unique tourism resources. Simultaneously, the integrated technical proposal of less investment and short development cycle sets up a new style of e-tourism.

\section{ACKNOWLEDGMENT}

This study was supported by the National Key Research and Development Plan of China (Grant No. 2016YFC0502606) and the Guizhou Technology Development plan, the Science and Technology Program of Guizhou (Grant No. QKHSYZ[2014]3073) and the Science and Technology Program of Guizhou (Grant No. QKHGZ[2014]4004). The authors would like to thank Ms. Jia Min, a member of the Mountain Resources Research Center for translating this article from Chinese.

\section{REFERENCES}

[1] J.K. Cao, R.Q. Zhou, and L.H. Wu, "Hainan virtual tourism GIS based on speech interface," in Ecosystem Assessment and Fuzzy Systems Management, 2014, pp. 315-324.

[2] A.A. Wirjono, and Z.R. Lincoln, "Information visualization for tourist 
and travelling in Indonesia,” in International Conference on Advances in Computing and Communications, pp. 130-137, July 2011.

[3] A. Marcus, The Travel Machine: Combining Information Design. Research and design innovations for mobile user experience, 2013, pp. 261-357.

[4] J.P. Hobson, and A.P. Williams, "Virtual reality: a new horizon for the tourism industry,” in Journal of vacation marketing, 1(2), 1995, pp.124135.

[5] H. Berger, M. Dittenbach, D. Merkl, A. Bogdanovych, S. Simoff and C. Sierra, "Opening new dimensions for e-Tourism," in Virtual Reality, 11(2-3), 2007, pp. 75-87.

[6] Y. Xu, The design and implementation of tourism management system based on the SOA structure, Master's thesis, Nanjing University of Science and Technology, 2011.

[7] Z.N. Guan, Design and application of the online travel products system basing on .NET, Master's thesis, Jilin University, 2014.

[8] Z.H. Dong, The design and implementation of digital tourism system based on B/S structure, Doctoral dissertation, Henan University, 2006.

[9] D. Zhao, Design and implementation tourism information system based on WebGIS, Master's thesis, Yangtze University, 2015.

[10] L. Yang, Research on the development and application of virtual tourism, Master's thesis, Shanghai Jiao Tong University, 2011.

[11] Y. Lu, Design and implementation of virtual tourism system, Master's thesis, Northwestern Polytechnical University, 2006.

[12] J.D. Zhang, Design of virtual tourism system based on WebGIS, Master's thesis, Jilin University, 2006.

[13] W.Y. Jiang, R.P. LUAN, "Design and realization of virtual tourist landscape based on VRML ArcGIS,” in Geographical Research, vol. 29, pp. 1715-1723.

[14] Y.S. Wei, The design and implementation of panorama 3D tour based on WebGL, Master's thesis, Nanjing University, 2016.

[15] T.R. Gao, J.P. Xiao, P.S. Peng, P. Fang, and T. Liu, "Research on Virtual Landscape System," in Urban Geotechnical Investigation \& Surveying, vol. 1, pp. 25-27. 